\title{
Temporal Trends of Cardiac and Respiratory Responses to Ventilatory Challenges in Congenital Central Hypoventilation Syndrome
}

\author{
PAUL M. MACEY, CLAIRE VALDERAMA, AMY H. KIM, MARY A. WOO, DAVID GOZAL, \\ THOMAS G. KEENS, REBECCA K. HARPER, AND RONALD M. HARPER \\ Departments of Neurobiology [P.M.M., C.V., A.H.K., R.K.H., R.M.H.], School of Nursing [M.A.W.], and \\ the Brain Research Institute [R.M.H.], David Geffen School of Medicine at UCLA, University of \\ California at Los Angeles, Los Angeles, California 90095, U.S.A.; Department of Pediatrics, University of \\ Louisville School of Medicine, Louisville, Kentucky 40202, U.S.A. [D.G.]; Childrens Hospital Los Angeles, \\ Los Angeles, California 90027, U.S.A. [T.G.K.]
}

\begin{abstract}
Congenital central hypoventilation syndrome (CCHS) patients exhibit respiratory deficits to ventilatory challenges, diminished breathing drive during sleep, and reduction of respiratoryrelated heart rate variation, but at least partially preserved peripheral chemoreception. We hypothesized that integration of afferent activity with respiratory motor output is deficient in CCHS, rather than chemoreceptor failure, and that examination of trends in heart and breathing rates and variabilities following ventilatory challenges may clarify the deficient mechanisms. Twelve children with CCHS and 12 age- and gender-matched control cases were subjected to hyperoxic hypercapnic, poikylocapnic hypoxic, and hyperoxic challenges while supine. Heart and respiratory rates and variabilities during 60-s baseline and 120 -s challenge periods were assessed. Hypoxia and hypercapnia enhanced breathing rate in control subjects; in CCHS cases, the rise differed during hypercapnia and did not occur to hypoxia. Hyperoxia showed initial transient patterns in breathing rate that differed between groups. A heart rate increase to hypoxia and late decline to hyperoxia were muted in CCHS patients. In hypercap-
\end{abstract}

\section{ABSTRACT}

nia, heart rate followed similar rising patterns in both groups. Overall CCHS heart rate variability was lower in baseline and challenge periods, principally due to diminished respiratoryrelated variation, especially during hypercapnia. No heart rate variability group differences emerged in hypoxia, and only a late increase for CCHS cases developed in hyperoxia. The findings indicate retention of aspects of chemoreceptor sensitivity in CCHS cases. The heart rate alterations to ventilatory challenges suggest specific compensatory responses of a slower nature remain intact in CCHS, whereas other rapidly changing components are deficient. (Pediatr Res 55: 953-959, 2004)
Abbreviations
CCHS, congenital central hypoventilation syndrome
ECG, electrocardiographic
ET $_{\mathbf{C O} 2}$, end-tidal $\mathrm{CO}_{2}$
fMRI, functional magnetic resonance imaging
RMANOVA, repeated measures ANOVA
RSA, respiratory sinus arrhythmia

A number of alterations in respiratory and cardiovascular control characterize CCHS (also known as "Ondine's curse"), including diminished ventilatory responses to $\mathrm{CO}_{2}$ and hypoxia (1-4), a reduced drive to breathe during sleep (5), greatly diminished high frequency contributions) to heart rate variability $(6,7)$, and abolition of cold pressor-induced respiratory changes (8). Despite these deficiencies, other respiratory and cardiovascular functions remain intact. CCHS patients can increase ventilation voluntarily in response to exercise, and to

Received May 22, 2003; accepted December 9, 2003.

Correspondence: R.M. Harper, Ph.D., Department of Neurobiology, David Geffen School of Medicine at UCLA, University of California at Los Angeles, Los Angeles, CA 90095-1763, USA; e-mail: rharper@ucla.edu

Supported by National Institute of Child Health and Human Development HD-22695.

DOI: 10.1203/01.PDR.0000125262.82405.3C passive motion of the extremities, even during sleep (9-12). Preservation of cardiovascular-related patterns include the slower heart rate variation induced by cold pressor stimulation, or induced by slower respiratory efforts accompanying Valsalva maneuvers (8). In addition, the spontaneous slow variation of heart rate that normally occurs in response to multiple sources during sleep is also present $(5,7)$, despite loss of faster respiratory-related influences.

The classical description of CCHS focuses on central chemoreceptor dysfunction $(5,13)$, however, components of both peripheral and central chemoreception appear to remain at least partially intact in CCHS. Respiratory rates increase in response to hypoxia and decline when subjects are exposed to hyperoxia $(3,14)$. Both children with CCHS and adults with central hypoventilation syndrome surgically induced by resection of 
medullary tumors arouse from sleep to hypercapnic stimulation $(15,16)$. Collectively, the evidence suggests retention of at least components of chemoreceptor afferent activity, that central components underlying respiratory motor output may be less affected, and that specialized deficits occur in the integration of sensory afferent inputs with breathing outputs, as previously proposed (3).

We hypothesized that integration of afferent activity with respiratory motor output remains the central deficit in CCHS, that this deficiency principally affects more rapidly changing components of breathing control, and that these processes can be revealed by examining rapid and slow cardiovascular and respiratory responses to ventilatory challenges. Particular challenges, such as hypercapnia which enhances respiratory sinus arrhythmia and variations in oxygen level, which alter heart rate variation at slower frequencies (17), should differentiate cardiovascular and respiratory responses in CCHS cases over controls and provide insights into deficient mechanisms.

We assessed the time course of heart and respiratory rates and variabilities to hypercapnic, hypoxic, and hyperoxic challenges with the objective of delineating failing central neural mechanisms that mediate these challenges in CCHS. Evaluation of the time course of responses to challenges was used to partition peripheral and central chemoreceptor action, as well as to determine longer-term cardiac and respiratory patterns.

\section{METHODS}

Subjects. Fourteen children with a diagnosis of CCHS and 15 control subjects participated in the study. Due to technical data-acquisition issues (lost or artifact-contaminated signals), a subset of 12 age- and gender-matched children from each group was used for the analysis (10 for heart rate during hypoxia). The mean age $\pm \mathrm{SD}$ (range) was $10.9 \pm 1.9$ (8-14) $\mathrm{y}$ for control subjects and $10.9 \pm 2.4(8-15)$ y for CCHS cases. Standard criteria (18) were used to establish the diagnosis of CCHS, and included ventilator dependency during sleep but not during wakefulness; absence of primary pulmonary, cardiac, or neuromuscular disease; and markedly reduced ventilatory responses to hypercapnia. We excluded CCHS cases with concomitant Hirschsprung's disease to avoid potential confounds from aberrant neural innervation to the viscera. All CCHS cases received a tracheostomy early in life for nighttime ventilation, however, these openings were occluded during waking hours and during the challenges. No control subjects had been subjected to this procedure. Physiologic assessments were made concurrently with fMRI procedures, so that brain structures involved in the ventilatory challenges could be visualized; those visualization studies will be reported separately. For all challenges, subjects lay supine in an MRI scanner. Baseline recordings were collected with subjects breathing room air for $60 \mathrm{~s}$, after which the gas mixtures, hyperoxic hypercapnia $\left(5 \% \mathrm{CO}_{2}\right.$ in $\left.95 \% \mathrm{O}_{2}\right)$, poikylocapnic hypoxia $\left(15 \% \mathrm{O}_{2}, 85 \% \mathrm{~N}_{2}\right)$, or hyperoxia $\left(100 \% \mathrm{O}_{2}\right)$ were administered, each lasting $120 \mathrm{~s}$, and separated by a minimum of $8 \mathrm{~min}$

Data acquisition and analysis. $\mathrm{ECG}$ and relative $\mathrm{ET}_{\mathrm{CO} 2}$ measures were collected and stored. Digitizing rates for ECG signals were $1 \mathrm{kHz}$, and $64 \mathrm{~Hz}$ for $\mathrm{ET}_{\mathrm{CO} 2}$. Cardiac RR intervals were measured from the ECG signal. Respiratory frequency and variability measures were derived from the $\mathrm{ET}_{\mathrm{CO} 2}$ signal. Automatic peak-trough detection software was used to determine inspiratory and expiratory onset times. Detected peaks were reviewed on a digital display, which included raw signals and computer-selected inspiratory times, by an experienced observer who could evaluate and correct artifactual or erroneous detections, based on the context of other physiologic signals.

Cardiac intervals were plotted during the baseline and challenge conditions and compared, with RMANOVA, to evaluate group differences in heart rate and breathing rate during the recording periods, with comparisons between baseline and challenge for each group, and between groups during the challenge (19). A significance threshold of 0.05 was adopted. The challenge was divided into 1210 -s epochs, and the Tukey-Fisher criterion for multiple comparisons was used before testing for significance at individual epochs. Raw cardiac beat-to-beat intervals were successively plotted to optimally visualize momentary changes in variation and to display short-term and long-term patterns in heart rate. A measure of variation attributable to breathing, RSA, was determined by evaluating the extent of heart rate change per breath. For each breath, the corresponding trough-to-peak heart rate increase during and after inspiration and the magnitude of the subsequent peak-to-trough heart rate decrease corresponding to expiration were averaged to give the RSA for that breath. These values were assessed by RMANOVA and averaged over subjects within each group for display. Nonstationary aspects of the cardiac rate changes precluded assessment of variation with spectral analysis techniques.

In a subset of subjects (control $n=10$ and CCHS $n=7$ ), $\mathrm{ET}_{\mathrm{CO} 2}$ was recorded during baseline and challenge conditions.

This study was approved by the institutional committee on human research; informed written consents were obtained from the subjects' caregivers.

\section{RESULTS}

Baseline. Mean baseline heart rate for control subjects $( \pm \mathrm{SD})$ was $89.2 \pm 12.6$ beats $/ \mathrm{min}$, and for CCHS was $91.2 \pm$ 12.9 beats/min (NS), as shown in Table 1; baseline respiratory frequency was $17.1 \pm 4.7$ breaths/min and $19.2 \pm 3.7$ breaths/ min for control and CCHS subjects, respectively (NS). Variability (interquartile range) of heart rate for control subjects was 9.3 beats $/ \mathrm{min}$ and 5.2 beats $/ \mathrm{min}$ for CCHS cases $(p<$ $0.05)$; respiratory rate variability for control subjects was 5.1 breaths $/ \mathrm{min}$ and 4.3 breaths/min for CCHS cases (NS). In subsets of control and CCHS subjects, $\mathrm{ET}_{\mathrm{CO} 2}$ was higher during baseline and challenge periods in CCHS cases (Table 1). $\mathrm{ET}_{\mathrm{CO} 2}$ was higher during hypercapnia challenges in both groups ( $t$ test, $p<0.05$ ), but was unchanged to hypoxia or hyperoxia.

Overall heart and respiratory rate variability. An overall diminished absolute heart rate variability in CCHS cases was apparent both during baseline and during all respiratory challenges. The reduced variability derived primarily from loss of 
Table 1. Control and CCHS subjects' respiratory rate (BR), heart rate (HR), and, for a subset of subjects, ET $T_{C O 2}$ values averaged during 2-min challenge periods, and during baseline periods ( \pm SD across subjects)

\begin{tabular}{|c|c|c|c|c|c|c|}
\hline & \multicolumn{2}{|c|}{$\mathrm{BR}$} & \multicolumn{2}{|c|}{ HR } & \multicolumn{2}{|c|}{$\mathrm{ET}_{\mathrm{CO} 2}$} \\
\hline & Control & CCHS & Control & CCHS & Control $(n=10)$ & CCHS $(n=7)$ \\
\hline Baseline & $17.1 \pm 4.7$ & $19.2 \pm 3.7$ & $89.2 \pm 12.6$ & $91.2 \pm 12.9$ & $37.8 \pm 5$ & $53 \pm 5.7$ \\
\hline Hypercapnia & $18.5 \pm 5.2$ & $19.2 \pm 4.3$ & $91.5 \pm 18.6$ & $90.9 \pm 12.6$ & $41.6 \pm 3.1$ & $56.4 \pm 6.9$ \\
\hline Hypoxia & $17 \pm 5.4$ & $19.7 \pm 4.1$ & $89.8 \pm 9.7$ & $93.6 \pm 14$ & $37.5 \pm 3.5$ & $52.4 \pm 4.8$ \\
\hline Hyperoxia & $19 \pm 8$ & $19.4 \pm 4.9$ & $90 \pm 18.4$ & $92.4 \pm 13.2$ & $36 \pm 4$ & $49.2 \pm 3.3$ \\
\hline
\end{tabular}

more-rapid (principally respiratory-related) changes in heart rate, as displayed in the raw RR interval plots (Fig. 1) and in the average RSA plots (Fig. 2). Respiratory variability differences between groups were apparent during each challenge (Fig. 3).

Hypercapnia. In both groups, heart rates increased shortly after the challenge onset to $\mathrm{CO}_{2}$ ( $p<0.05$, Fig. 4). The rise in both groups was biphasic, with a decline in cardiac frequency that emerged 30-40 s after onset of the hypercapnic stimulus. However, $60 \mathrm{~s}$ after hypercapnic challenge onset, CCHS cardiac rates increased again, whereas control subjects' rates remained low; toward the end of the challenge, heart rates increased in both control and CCHS groups. Mean heart rates were similar in both groups (Table 1). However, cardiac variability was enhanced in control subjects during the course of $\mathrm{CO}_{2}$ administration (Fig. 3). This increase in variability primarily resulted from the much more prominent respiratoryrelated variation induced by hypercapnia in control subjects

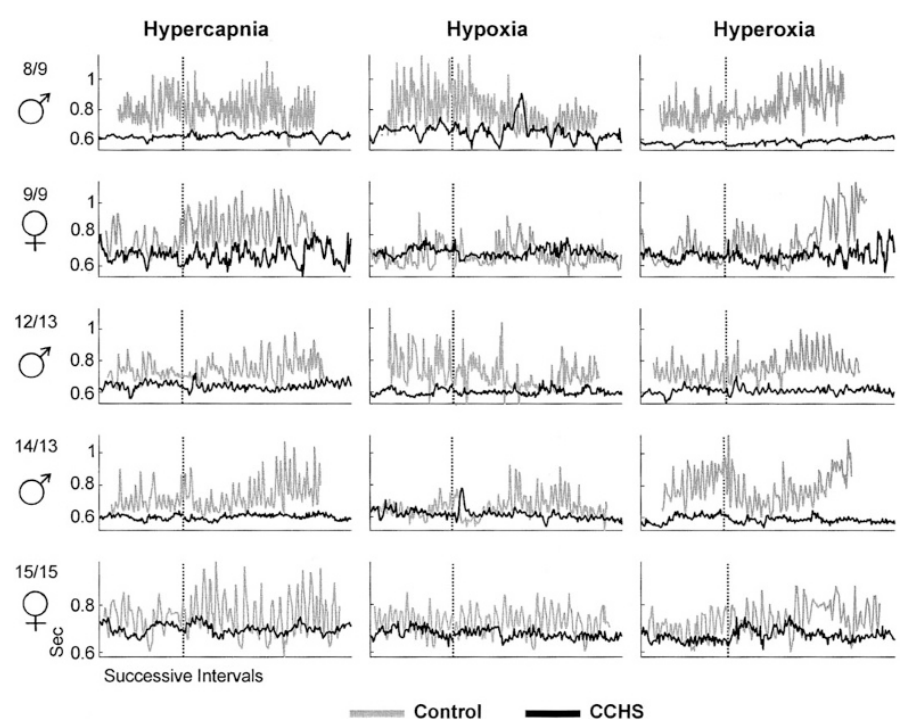

Figure 1. Plots of successive cardiac RR (interbeat) intervals, with interbeat interval period represented on the $y$ axis (in seconds), and count of interval represented on the $x$ axis for five typical individual control subjects (gray traces) and five age- and gender-matched CCHS cases (black traces) during the 60 -s baseline and 120 -s ventilatory challenges. Gender for each matched pair is indicated to the left of the hypercapnia $y$ axis as are the ages, in years, for subjects in each pair of control/CCHS subjects. Onset of challenge is indicated by the vertical dashed line in each trace. The length of each trace varies, because the successive number of intervals is represented on the $x$ axis (rather than time); shorter intervals (i.e., faster heart rates), being more numerous for the fixed time period of $150 \mathrm{~s}$, are represented by longer traces. Hypercapnia (hyperoxic hypercapnia: $3 \% \mathrm{CO}_{2}, 95 \% \mathrm{O}_{2}$ ); Hypoxia (poikylocapnic hypoxia: $15 \% \mathrm{O}_{2}$, balance, $\left.\mathrm{N}_{2}\right)$; and Hyperoxia $\left(100 \% \mathrm{O}_{2}\right)$.
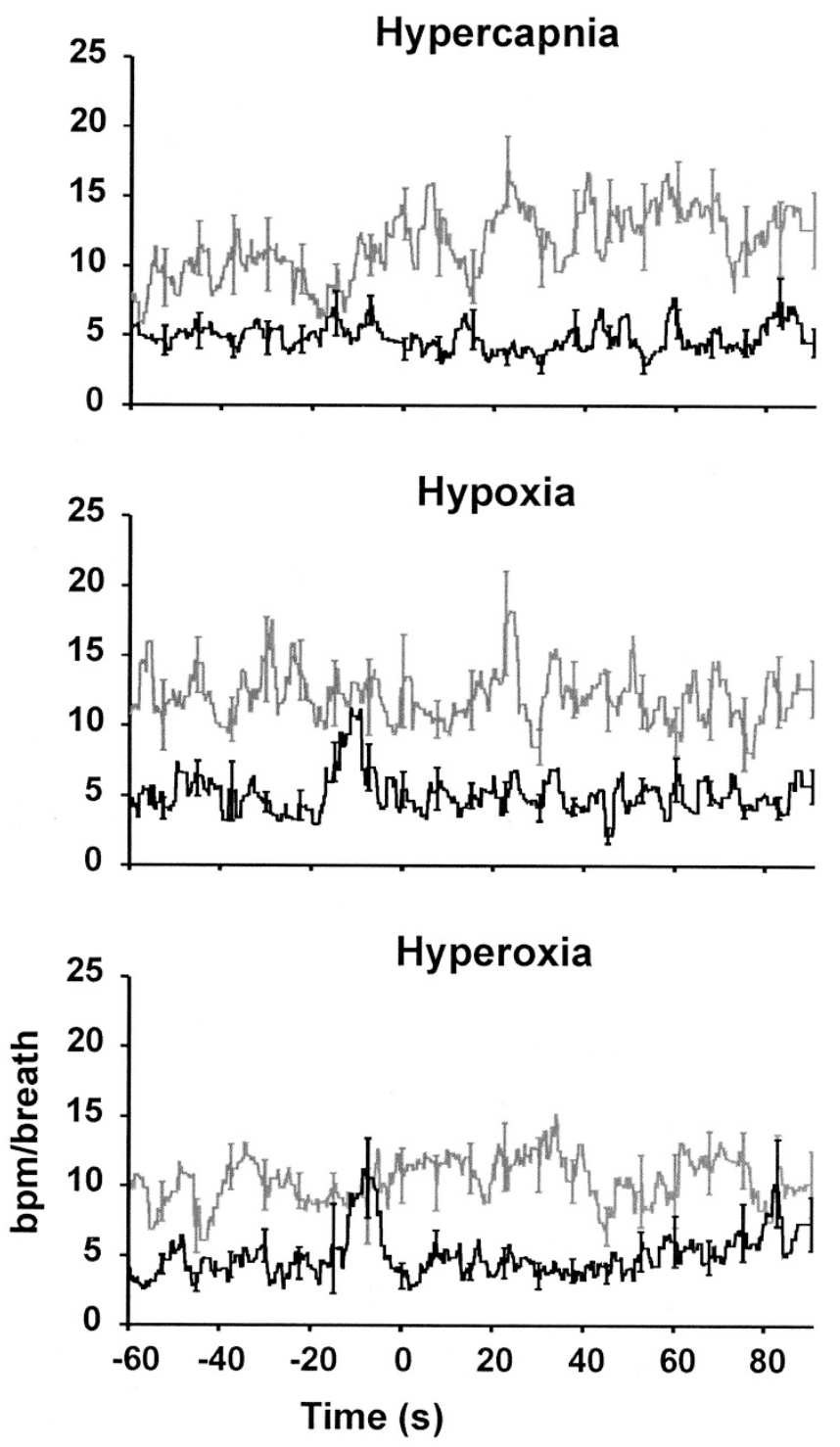

- CCHS - Control

Figure 2. Average RSA values, with between-subject SE bars, for all CCHS and control cases are partitioned from other sources of variability and plotted over the course of each ventilatory challenge as variation in cardiac beats $/ \mathrm{min}$ (bpm) per breath.

(Fig. 2); slower trends also contributed (Figs. 1, 2, and 5). Only modest changes occurred in CCHS patients.

Hypoxia. Control subjects increased respiratory rates to hypoxia late (60 s) in the challenge, but rates did not rise in CCHS cases (Fig. 4). Heart rates increased to hypoxia in both groups; the increase was less pronounced in CCHS than control 


\section{Breathing Rate Variability (breath/min) \\ Heart Rate Variability (beat/min)}
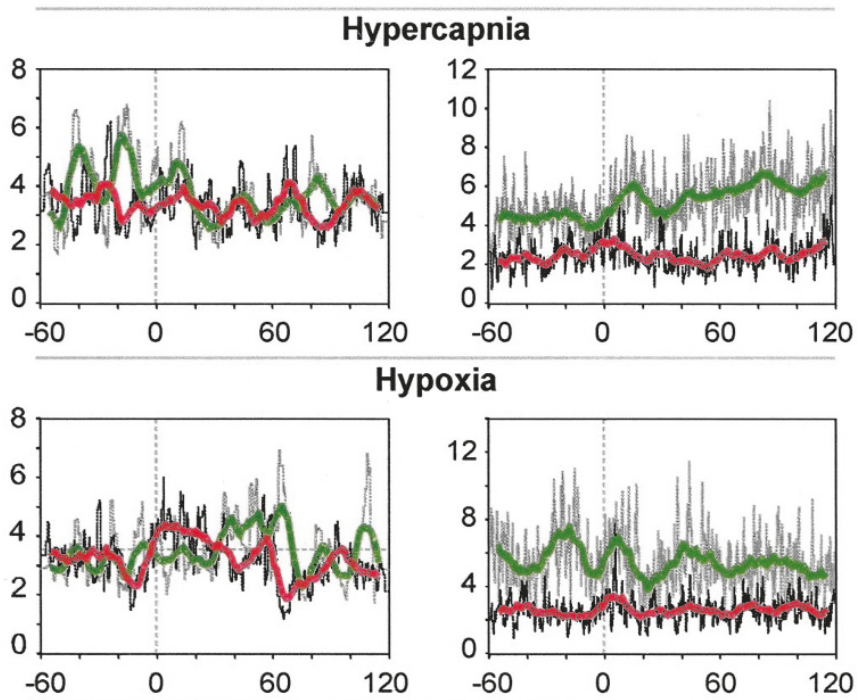

Hyperoxia
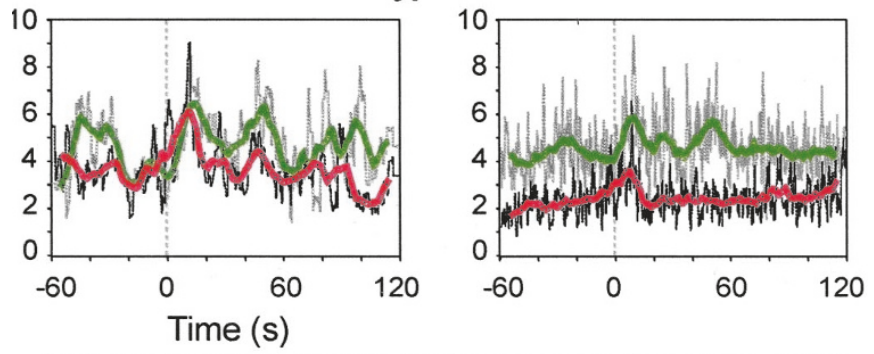

Variability curves
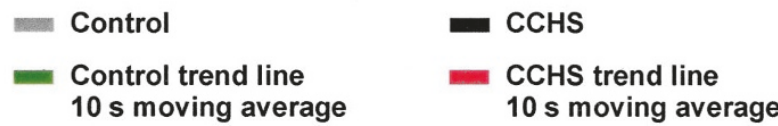

Figure 3. Average breathing and heart rate variabilities, together with $10-\mathrm{s}$ moving average trend lines during the three challenges, with black traces representing CCHS case values and gray traces representing control subject values. Ten-second moving average trend lines are drawn for each group (CCHS, red; control, green). Challenge onset is at time $0 \mathrm{~s}$ (vertical dashed line), after $60 \mathrm{~s}$ of room air baseline.

subjects. No differences in change in heart rate variability were found between groups (Fig. 5). Respiratory variability increased in control subjects concurrently with the rise in breathing rate; CCHS cases showed a rise in respiratory variability only early in the challenge.

Hyperoxia. A momentary increase in respiratory rate developed during the first $10 \mathrm{~s}$ of the challenge in response to hyperoxia in control subjects (Fig. 4). The CCHS cases showed a comparable transient effect in the opposite direction. Respiratory rates later declined in both groups, but only transiently in control subjects. Heart rate initially increased, but later declined in both groups. The extent of increase and decrease in heart rate was significantly greater in control than in CCHS cases. Respiratory variability was higher during hyperoxia in control than in CCHS cases, but heart rate variability showed only a transient small increase, comparable across both groups, with the groups differing only at the end of the challenge (Fig. $5)$.
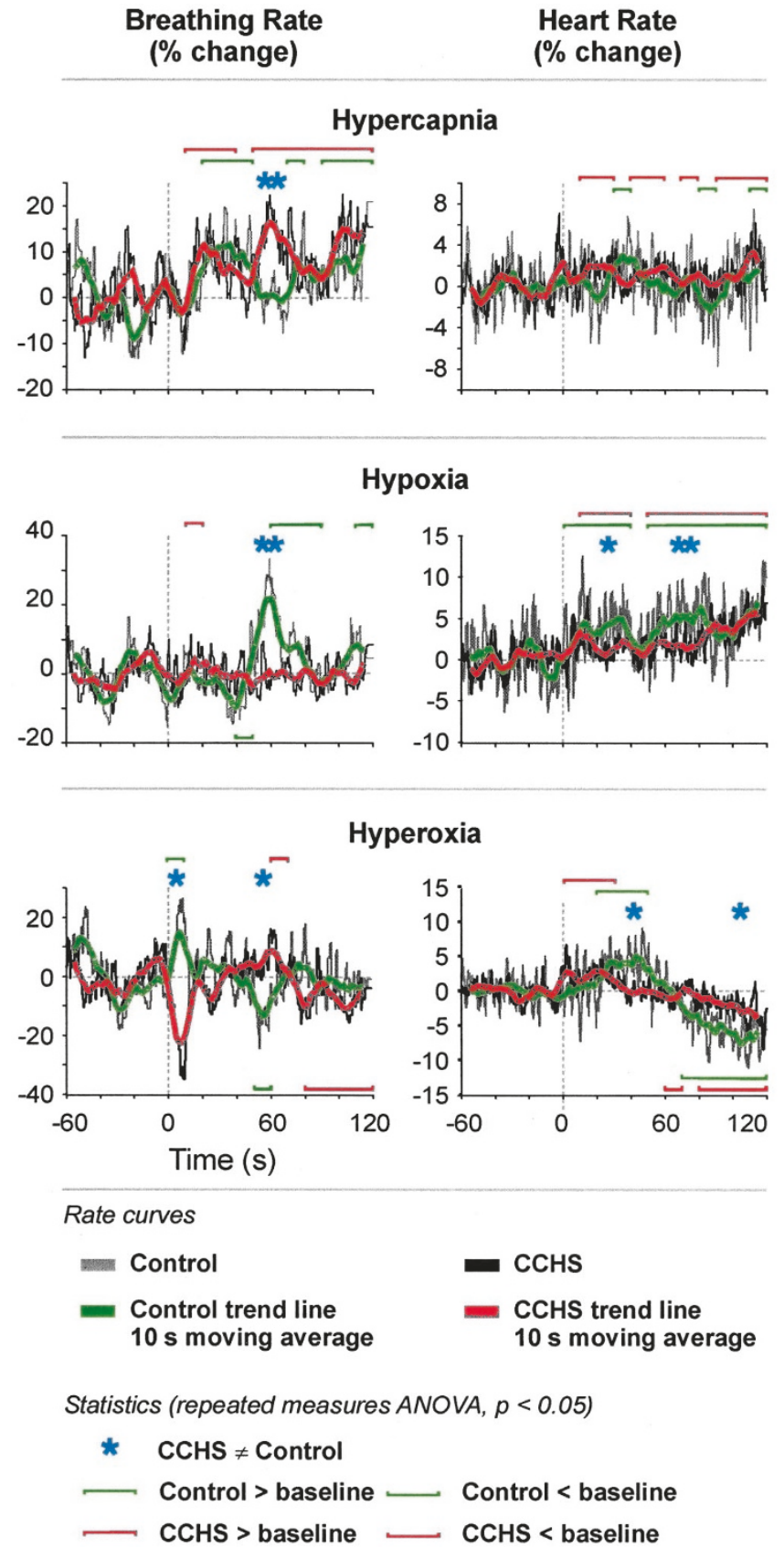

Figure 4. Traces of averaged percentage change in breathing and heart rates, together with trend lines, over baseline and during hypercapnic, hypoxic, and hyperoxic challenges, with color coding of traces and starting point indications as in Figure 3. Significant changes from baseline are represented in colored lines above or below the traces over relevant epochs (red, CCHS; green, control); significant differences between groups are indicated by blue asterisks, similarly placed over appropriate epochs.

\section{DISCUSSION}

A marked similarity of change in heart rate patterns emerged in both groups to the challenges, despite the large overall difference in heart rate variability. CCHS cases, however, showed substantially reduced rapid, respiratory-related cardiac variation during baseline and during challenges, and especially during hypercapnia, which enhanced RSA in control cases. Distinct respiratory patterns also emerged, particularly during hypoxia, with the CCHS patients not showing the expected rise 


\section{Breathing Rate Variability} (\% change)

\section{Heart Rate Variability} (\% change)

Hypercapnia

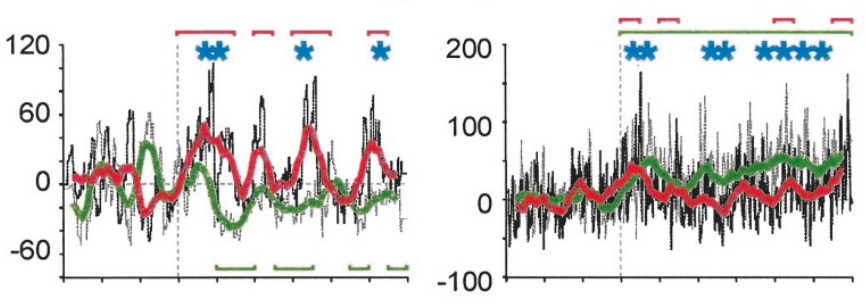

\section{Hypoxia}
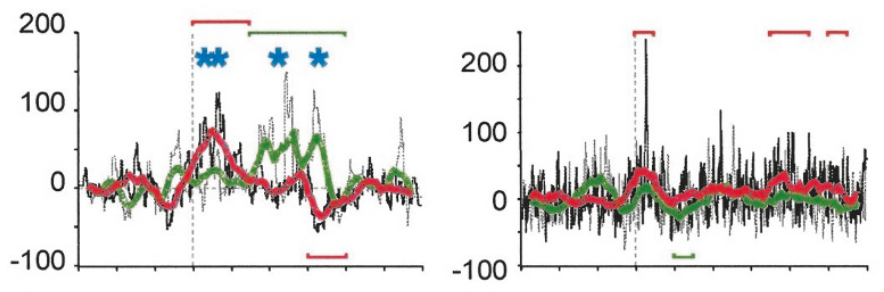

Hyperoxia

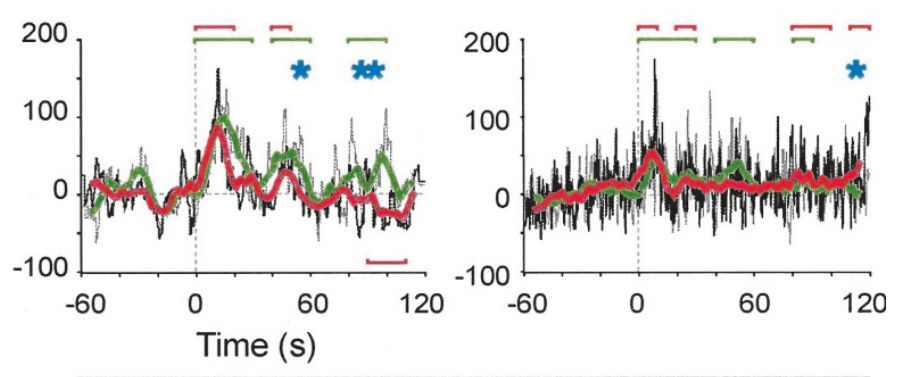

Variability curves.
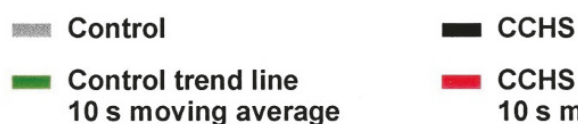

10 s moving average

- CCHS trend line $10 \mathrm{~s}$ moving average

Statistics (repeated measures ANOVA, $p<0.05$ )

\section{* $\mathrm{CCHS} \neq$ Control}

Control $>$ baseline

\section{Control $<$ baseline}

CCHS > baseline

CCHS < baseline

Figure 5. Average percentage change in breathing and heart rate variabilities (in percentage change from baseline) over each baseline and challenge period, with color-coding of traces, trend lines, epochs of significant effects, and start time indications as in Figure 4.

in breathing rate. However, although the time course of respiratory responses to hypercapnia in CCHS cases differed, breathing rates rose in both groups, and CCHS heart rates followed similar patterns to those of control subjects throughout the $120 \mathrm{~s}$ challenge period. The respiratory and cardiac rate responses to the ventilatory challenges reinforce evidence suggesting that CCHS respiratory deficits cannot be interpreted as a simple failure of central or peripheral chemoreception.

Although CCHS patients showed transient early respiratory rate differences from control subjects to hyperoxia, heart rate declined in a similar fashion, with only momentary pattern differences. Moreover, although CCHS cases did not respond appropriately with respiratory rate adjustments to hypoxia, heart rates rose in a manner similar to the control subjects, even if more slowly. The findings suggest a degree of sensitivity to low oxygen and some retention of appropriate components of the cardiovascular response. Furthermore, although the time course of respiratory responses to hypercapnia in CCHS cases differed, breathing rates rose in both groups, and CCHS heart rates followed similar patterns to those of control subjects throughout the 120 -s challenge period. The heart rate responses, together with current and previously demonstrated respiratory responses to cold pressor and volitional breathing exercises, suggest that deficient processes in CCHS ventilatory control are largely separate from slower integrative components and voluntary influences, and that these deficiencies relate principally to rapid integration of stimuli, resulting in abnormal respiratory musculature output.

Diminished overall variation in cardiac rates for CCHS cases was apparent in all baseline conditions, and the substantial differences in overall variability remained unchanged after administration of all challenges. Heart rate variability, principally from respiratory-related sources, increased even further in control subjects over the course of the hypercapnic challenge; the enhanced RSA to hypercapnia has been previously demonstrated by others in control subjects (20), and appears to be a mechanism to increase the efficiency of pulmonary circulation $(21,22)$. The failure of CCHS cases to increase RSA to hypercapnia represents one more deficit in central chemoreceptor integration with appropriate cardiovascular action (23). A reduction of RSA under resting conditions in CCHS has been a consistent finding in baseline recordings, particularly during sleep $(6,7)$, and after pressor challenge conditions (8).

Peripheral oxygen afferent processing. An initial transitory respiratory rate response emerged to hyperoxia in both control and CCHS cases, but the direction of these responses differed, with an increase in control cases and a decrease in CCHS patients. The underlying mechanism of the transitory responses to elevated oxygen tension is unclear. The transient responses occurred within $10 \mathrm{~s}$, and were at the time limits of mediation by carotid body receptors. Increased concentrations of inspired $\mathrm{CO}_{2}$ can stimulate $\mathrm{CO}_{2}$ or $\mathrm{pH}$ sensors in the upper airway and mucosa independently of the peripheral chemoreceptors; this afferent activity is mediated by the superior laryngeal nerve (24). Comparable stimulation of upper airway chemosensory pathways in humans has been indicated by others (25), and suggests the potential for modification of respiratory patterns by airway stimulation. Thus, the immediate transient responses could have resulted from a neural reflexogenic response to inactivation of intra-airway peripheral chemoreceptor sensors in control cases (26), the latter sensors being dysfunctional in CCHS (27).

The later respiratory and cardiovascular responses to hypoxia and hyperoxia were of particular interest. Hyperoxia, through suppression of tonic peripheral chemoreceptor activity, would normally lead to a reduction in ventilation, whereas hypoxia would usually lead to enhanced ventilatory output by stimulating these chemoreceptors. Although CCHS patients, unlike control subjects, did not increase breathing rates to the 
hypoxic challenge, heart rates responded near normally. Because CCHS cases responded with near-adequate respiratory and heart rate changes to hyperoxia, it is likely that peripheral chemoreceptor functions are partially preserved, as previously indicated (14). The current findings suggest the ability, in CCHS cases, to induce suppression of tonic chemoreceptor action, such that the response to hyperoxia appears to be appropriately mediated $(3,14)$. Hypoxia is normally accompanied by increased peripheral chemoreceptor afferent activity (28). In contrast to hyperoxic responses, the failure to appropriately increase respiratory rates to hypoxia in CCHS cases suggests deficits of some component of the respiratory control circuit. However, the relatively rapid adjustment of heart rate to hypoxia further indicates adequate afferent input from the periphery, and successful integration of those inputs within neural cardiovascular system pathways. Thus, it is likely that particular brainstem or even more rostral brain regions that preferentially relay processes for integrative respiratory activity are the regions that are most affected in CCHS, a possibility that is supported by the classical phenotypic manifestations of the disorder.

Trigeminal and voluntary influences. Stimulation of trigeminal afferents mediating certain sensory-related inputs results in different respiratory response patterns in CCHS compared with control children. For example, cold pressor application to the forehead enhances respiratory efforts in control but not in CCHS subjects (8). Our challenges bypassed trigeminal sensors of the nasal passages, because the subjects wore nose clips, but trigeminal mediation of stimulation of the oropharynx was still a possibility. The issue of airway reflexogenic stimulation needs to be further explored in CCHS. Low-frequency heart rate patterns associated with exaggerated voluntary expiratory efforts or appearing with forehead cold pressor challenges are retained in CCHS (8), even when faster variation from spontaneous breathing is diminished. Thus, ventilatory and reflexive cold pressor responses of a slow nature or of a voluntary characteristic appear to be retained in CCHS, whereas rapidly changing aspects of trigeminal influences and selective components of hypoxic and hypercapnicrelated responses are markedly reduced.

Neural mechanisms. Because voluntary breathing efforts are relatively unaffected in CCHS (8), it is unlikely that ventral frontal cortical areas, which regulate the upper airway and show damage in other breathing disorders (29), or brainstem projections from that region are affected. Because peripheral chemoreception is at least partially retained, afferent projections to the nucleus of the solitary tract are likely intact. Afferent proprioceptive activity from the lower limbs likely contributes to respiratory enhancement in CCHS during exercise, inasmuch as passive cycling of the foot during sleep reestablishes ventilation (9). The most likely integrative site for that stimulation lies in cerebellar deep nuclei, influenced by overlying cerebellar cortex, which receives proprioceptive information to coordinate motor output. Peripheral limb motion accompanying exercise has long been known to couple with respiratory efforts $(30,31)$, and proprioceptive information plays an essential role in that coordination (32). The cerebellar fastigial nucleus shows significant responses to $\mathrm{CO}_{2}$ exposure
(33) and plays a major role in vagally mediated respiratory reflexes (34); damage to the nucleus results in an inability to cope with extremes of blood pressure changes (35). We speculate that damage to cerebellar fastigial nuclei, or to overlying cortical projections to these nuclei, leads to the impairment of rapid cardiac responses in CCHS to hypercapnia and hypoxia shown here, and the diminished drive to breathe that occurs during sleep. The means by which the fastigial nucleus might restore breathing during sleep in CCHS through cyclic leg movements are speculative, but may result from exaggerated proprioceptor activity to cerebellar cortex or deep nuclei arising from that movement. Restoration of regularity in breathing in normal infants can be accomplished by other cerebellar input, e.g. vestibular action through rocking (36).

Future studies. The possibilities that rapid-coordination of breathing efforts with cardiovascular action is mediated by cerebellar structures, and that damage to these structures results in CCHS characteristics, are readily testable. Cerebellar structures show functional responses to hypercapnic challenges, and respond to loaded breathing or pressor challenges on fMRI examination $(37,38)$. Structures that are normally recruited to ventilatory challenges in the cerebellum show functional deficits in preliminary studies of CCHS cases (37, 39). Examination of cerebellar neural responses to ventilatory challenges following increased proprioceptive drive in CCHS cases should show compensatory enhancement of those neural responses, as well as more-appropriate physiologic responses. Damaged cerebellar cortical projections to fastigial nuclei in a chronic (i.e. drug-free) animal preparation should show a loss of drive to breathe during sleep, a loss of respiratory-related heart rate variation, and impaired responses to blood pressure manipulation. Damage to this nucleus has been examined in anesthetized preparations (35), with findings of significant deficits to pressor challenges.

Limitations. These studies have several limitations. Respiratory rate contributes only a portion of minute ventilation, inasmuch as timing and amplitude of breathing are controlled by different mechanisms. CCHS patients tend to maintain a relatively constant tidal volume, and characteristically modify minute ventilation almost exclusively by changing respiratory rate (11). This pattern is in contrast with control subjects who modify minute ventilation by altering both respiratory frequency and tidal volume. These differences could play a role, albeit minor, in the cardiac and respiratory variability differences found between CCHS and control cases. In addition, as opposed to previous studies that were carried out in the upright or seated positions, these studies were conducted in the supine position for both groups. Body positional differences may modify both cardiovascular and respiratory response characteristics (40). This issue is of particular relevance to the cardiovascular measures, because blood pressure aspects are closely related to vestibular influences and other postural mechanisms (41). It is the case that all CCHS subjects breathed at night through a tracheostomy, unlike controls. Adaptation to reduced stimulation of the oral airway through loss of airflow during sleep may have altered afferent processes in CCHS cases to these challenges in the waking state. We consider that possibility not likely, because the tasks were administered 
during conditions when the tracheostomy was closed during the waking state, but the potential for such adaptation cannot be excluded.

\section{CONCLUSIONS}

The findings suggest that simplistic concepts of CCHS characteristics being attributed to deficits in central and peripheral chemoreceptor responsiveness are inadequate. Sloweracting cardiovascular changes, likely providing compensatory perfusion, remain largely intact in CCHS, whereas more rapidly changing influences on heart rate variability are severely affected. Although muted, the cardiac responses to hyperoxia, combined with selected near-adequate cardiac rate responses to hypoxia and hypercapnia, emphasize that respiratory/cardiac control in CCHS has unique, fast-response insufficiencies. Chemoreceptor sensitivity remains functional in CCHS; however, that sensitivity is not reflected in normal breathing or heart rate variability responses, and implicates integrative deficits, rather than sensory deficits per se.

\section{REFERENCES}

1. Commare MC, Francois B, Estournet B, Barois A 1993 Ondine's curse: a discussion of five cases. Neuropediatrics 24:313-318

2. Oren J, Kelly DH, Shannon DC 1987 Long-term follow-up of children with congenital central hypoventilation syndrome. Pediatrics 80:375-380

3. Paton JY, Swaminathan S, Sargent CW, Keens TG 1989 Hypoxic and hypercapnic ventilatory responses in awake children with congenital central hypoventilation syndrome. Am Rev Respir Dis 140:368-372

4. Weese-Mayer DE, Silvestri JM, Menzies LJ, Morrow-Kenny AS, Hunt CE, Hauptman SA 1992 Congenital central hypoventilation syndrome: diagnosis, management, and long-term outcome in thirty-two children. J Pediatr 120:381-387

5. Haddad GG, Mazza NM, Defendini R, Blanc WA, Driscoll JM, Epstein MA, Epstein RA, Mellins RB 1978 Congenital failure of automatic control of ventilation, gastrointestinal motility and heart rate. Medicine (Baltimore) 57:517-526

6. Silvestri JM, Hanna BD, Volgman AS, Jones PJ, Barnes SD, Weese-Mayer DE 2000 Cardiac rhythm disturbances among children with idiopathic congenital central hypoventilation syndrome. Pediatr Pulmonol 29:351-358

7. Woo MS, Woo MA, Gozal D, Jansen MT, Keens TG, Harper RM 1992 Heart rate variability in congenital central hypoventilation syndrome. Pediatr Res 31:291-296

8. Kim AH, Macey PM, Woo MA, Yu PL, Keens TG, Gozal D, Harper RM 2002 Cardiac responses to pressor challenges in congenital central hypoventilation syndrome. Somnologie 6:109-115

9. Gozal D, Simakajornboon N 2000 Passive motion of the extremities modifies alveola ventilation during sleep in patients with congenital central hypoventilation syndrome. Am J Respir Crit Care Med 162:1747-1751

10. Gozal D, Marcus CL, Ward SL, Keens TG 1996 Ventilatory responses to passive leg motion in children with congenital central hypoventilation syndrome. Am J Respir Crit Care Med 153:761-768

11. Paton JY, Swaminathan S, Sargent CW, Hawksworth A, Keens TG 1993 Ventilatory response to exercise in children with congenital central hypoventilation syndrome. Am Rev Respir Dis 147:1185-1191

12. Shea SA, Andres LP, Paydarfar D, Banzett RB, Shannon DC 1993 Effect of menta activity on breathing in congenital central hypoventilation syndrome. Respir Physiol 94:251-263

13. Shea SA 1997 Life without ventilatory chemosensitivity. Respir Physiol 110:199 210

14. Gozal D, Marcus CL, Shoseyov D, Keens TG 1993 Peripheral chemoreceptor function in children with the congenital central hypoventilation syndrome. J Appl Physiol 74:379-387
15. Marcus CL, Bautista DB, Amihyia A, Ward SL, Keens TG 1991 Hypercapneic arousal responses in children with congenital central hypoventilation syndrome. Pediatrics 88:993-998

16. Ayas NT, Brown R, Shea SA 2000 Hypercapnia can induce arousal from sleep in the absence of altered respiratory mechanoreception. Am J Respir Crit Care Med 162:1004-1008

17. Murasato Y, Hirakawa H, Harada Y, Nakamura T, Hayashida Y 1998 Effects of systemic hypoxia on R-R interval and blood pressure variabilities in conscious rats. Am J Physiol Heart Circ Physiol 275:H797-H804

18. American Thoracic Society 1999 Idiopathic congenital central hypoventilation syndrome: diagnosis and management. Am J Respir Crit Care Med 160:368-373

19. Littell RC, Milliken GA, Stroup WW, Wolfinger RD 1996 SAS System for Mixed Models. SAS Institute Inc., Cary, NC

20. Sasano N, Vesely AE, Hayano J, Sasano H, Somogyi R, Preiss D, Miyasaka K, Katsuya H, Iscoe S, Fisher JA 2002 Direct effect of $\mathrm{PaCO}_{2}$ on respiratory sinus arrhythmia in conscious humans. Am J Physiol Heart Circ Physiol 282:H973-H976

21. Hayano J, Yasuma F, Okada A, Mukai S, Fujinami T 1996 Respiratory sinus arrhythmia: a phenomenon improving pulmonary gas exchange and circulatory efficiency. Circulation 94:842-847

22. Giardino ND, Glenny RW, Borson S, Chan L 2003 Respiratory sinus arrhythmia is associated with efficiency of pulmonary gas exchange in healthy humans. Am J Physiol Heart Circ Physiol 284:H1585-H1591

23. Gozal D 2001 Central chemoreceptor function in children. Pediatr Pulmonol Supp 23:110-113

24. Bartlett D, Jr, Knuth SL 1992 Responses of laryngeal receptors to intralaryngeal $\mathrm{CO}_{2}$ in the cat. J Physiol 457:187-193

25. Berthon-Jones M, Sullivan CE 1984 Ventilation and arousal responses to hypercapnia in normal sleeping humans. J Appl Physiol 57:59-67

26. Youngson C, Nurse C, Yeger H, Cutz E 1993 Oxygen sensing in airway chemoreceptors. Nature 365:153-155

27. Cutz E, Ma TK, Perrin DG, Moore AM, Becker LE 1997 Peripheral chemoreceptors in congenital central hypoventilation syndrome. Am J Respir Crit Care Med 155:358363

28. Kara T, Narkiewicz K, Somers VK 2003 Chemoreflexes-physiology and clinical implications. Acta Physiol Scand 177:377-384

29. Macey PM, Henderson LA, Macey KE, Alger JR, Frysinger RC, Woo MA, Harper RK, Yan-Go FL, Harper RM 2002 Brain morphology associated with obstructive sleep apnea. Am J Respir Crit Care Med 166:1382-1387

30. Dejours P 1959 The regulation of ventilation during muscular exercise in man J Physiol (Paris) 51:929-935

31. Bechbache RR, Duffin J 1977 The entrainment of breathing frequency by exercise rhythm. J Physiol 272:553-561

32. Morin D, Viala D 2002 Coordinations of locomotor and respiratory rhythms in vitro are critically dependent on hindlimb sensory inputs. J Neurosci 22:4756-4765

33. Xu F, Frazier DT 1997 Respiratory-related neurons of the fastigial nucleus in response to chemical and mechanical challenges. J Appl Physiol 82:1177-1184

34. Xu F, Frazier DT 1997 Involvement of the fastigial nuclei in vagally mediated respiratory responses. J Appl Physiol 82:1853-1861

35. Lutherer LO, Lutherer BC, Dormer KJ, Janssen HF, Barnes CD 1983 Bilateral lesions of the fastigial nucleus prevent the recovery of blood pressure following hypotension induced by hemorrhage or administration of endotoxin. Brain Res 269:251-257

36. Tuck SJ, Monin P, Duvivier C, May T, Vert P 1982 Effect of a rocking bed on apnoea of prematurity. Arch Dis Child 57:475-477

37. Harper RM, Bandler R, Spriggs D, Alger JR 2000 Lateralized and widespread brain activation during transient blood pressure elevation revealed by magnetic resonance imaging. J Comp Neurol 417:195-204

38. Gozal D, Hathout GM, Kirlew KA, Tang H, Woo MS, Zhang J, Lufkin RB, Harper RM 1994 Localization of putative neural respiratory regions in the human by functional magnetic resonance imaging. J Appl Physiol 76:2076-2083

39. Spriggs D, Saeed MM, Alger JR, Woo MA, Woo MS, Gozal D, Keens TG, Harper RM 1999 Time course of functional magnetic resonance signal changes in response to hypercapnia in congenital central hypoventilation syndrome (CCHS). Abstr Soc Neurosci 25:280

40. Xie A, Takasaki Y, Popkin J, Orr D, Bradley TD 1993 Influence of body position on pressure and airflow generation during hypoxia and hypercapnia in man. J Physio 465:477-487

41. Yates BJ, Miller AD 1998 Physiological evidence that the vestibular system participates in autonomic and respiratory control. J Vestib Res 8:17-25 